\title{
Faecal Calprotectin as Reliable Non-invasive Marker to Assess the Severity of Mucosal Inflammation in Patients with Ulcerative Colitis
}

\author{
Mohamed N. El-khashab ${ }^{1}$, Salama Al goniemy ${ }^{1}$, \\ Ghada A. Salem ${ }^{1}$, Hisham I. Mostafa ${ }^{2}$ \\ ${ }^{1}$ Tropical Medicine Department, Faculty of Medicine, Zagazig Universiy, Egypt \\ ${ }^{2}$ Student Hospital, Zagazig University, Egypt.
} Corresponding Author
Mohamed N. El-

Khashab

Mobile:002012231619

41

E mail:

elkhashab2005@hotm ail.com

Received :14/9/2012

Accepted after

revision:10 /11 /2012

Key words:

Calprotectin;

ulcerative colitis,
Background and study aim: We aimed to evaluate the validity and accuracy of the faecal calprotectin in differentiating patients with IBD from those with IBS and in the assessment of the severity of intestinal mucosal inflammation in patients with ulcerative colitis (UC) which may facilitate in the prognosis and follow.

Patients and Methods: We studied 60 Patients who came to endoscopy unit with lower gastroenterological symptoms. Patients with history of infections, malignancy, gastrointestinal surgery, pregnancy, alcohol abuse or taking nonsteroidal anti-inflammatory drugs were excluded from study. All patients subjected to thorough medical history, simple clinical colitis activity index was determined with a score $>4$ indicate active UC, complete blood picture, liver, kidney function tests, ESR, CRP, ANCA were done, a stool sample for FC levels determined by a highly sensitive enzymelinked immunosorbent assay and total colonoscopy with histological examination of intestinal mucosa biopsy were done. The patients divided into 2 groups. Group A: patients with UC, group $\mathrm{B}$ : patients with manifestation of irritable bowel syndrome as a control group.

\section{INTRODUCTION}

The cause of ulcerative colitis (UC) is currently under examination. It is believed that the 2 idiopathic forms of inflammatory bowel disease (IBD), ulcerative colitis and Crohn's disease
Results: There was a high significant difference between individuals with no pathological activity and other degree of mucosal inflammation as regard simple clinical colitis activity index, endoscopic appearance and faecal calprotectin ( $\mathrm{p}=$ 0.000). The sensitivity, specificity, positive predictive value and negative predictive value of faecal calprotectin in diagnosis of UC were $93.5 \%, 89.7 \%$, $90.6 \%$, and $92.9 \%$ respectively. The positive predictive value and negative predictive value of simple clinical colitis activity index for diagnosis of UC were $76.5 \%$ and $80.8 \%$ respectively. The positive predictive value and negative predictive value of endoscopic appearance for diagnosis of UC were $100 \%$, and $85.3 \%$ respectively. There was a high significant difference and positive correlation between faecal calprotectin, score of colonic pathological activity, endoscopic appearance and simple clinical colitis activity index.

Conclusion: Faecal calprotectin is highly useful for the diagnosis and disease monitoring of patients with UC as it is easy, non invasive, reliable tool.

(CD), develop secondary to complex interactions among genetic predispositions, environmental risk factors, and the immune system. 
Several genes likely play a role; their products, when combined with environmental factors and dysfunctional immunity, result in a disease spectrum with heterogeneous manifestations and many unique phenotypes [1].

The determination of inflammatory activity is crucial for patients with IBD for the diagnosis, monitoring and step up of therapy. Colonoscopy is the accepted gold standard for investigation of the colon, but is invasive and associated with risks [2]. Among objective clinical features; bloody stool frequency, body temperature and heart rate are good predictors of outcome. Laboratory markers have been studied intensively with varying degrees of success. The widely used acute phase protein C-reactive protein in this respect is a less good marker for assessing disease activity in UC than Crohn's disease [3].

More recently, faecal markers have demonstrated promising results. The most studied markers are faecal calprotectin and lactoferrin have shown accuracy at detecting colonic inflammation [4].

Calprotectin is a calcium-binding protein that is derived predominantly from neutrophils and, to a lesser extent, from monocytes and reactive macrophages [5].

It is worth noting that fecal calprotectin concentrations correlate more closely with histological than macroscopic (endoscopic) findings, suggesting that this biological marker is more sensible than endoscopy in evaluating IBDs activity [6].

The present study aimed at evaluation of the accuracy of faecal calprotectin and correlate it with clinical scores, common serum markers and endoscopy in the assessment of the severity of intestinal mucosal inflammation in patients with ulcerative colitis.

\section{PATIENTS AND METHODS}

This present study was conducted in the Tropical medicine department and gastrointestinal endoscopy unit, faculty of medicine, Zagazig University during the period from January 2011 to March 2012.

Our study included 31 patients with ulcerative colitis. The control group comprised 29 patients with manifestation of IBS matched for age and sex with patient's group. Written informed consents were obtained prior to participation in this study.

Patients with history of infections (recent respiratory or urinary tract infections within 1 month), malignancy (current), gastrointestinal trauma or surgery (within 1 month), or regularly taking aspirin, anticoagulants, or non-steroidal anti-inflammatory drugs, pregnancy and history of alcohol abuse were excluded from this study.

All patients should be subjected to the following:

- Thorough medical history taking.

- Simple clinical colitis activity index (SCCAI).

- Thorough clinical examination.

- Complete blood picture.

- Liver and kidney functions tests.

- Blood sample for estimation of ESR, and of CRP.

- Determination of ANCA in serum.

- Thorough stool examination.

- Quantitative measurement of faecal calprotectin levels were measured by a highly sensitive enzyme-linked immunosorbent assay $\left(\mathrm{PhiCal}^{\mathrm{TM}}\right)$.

- Total colonoscopy with histological examination of intestinal biopsy specimens.

\section{Statistical analysis:}

Comparisons between means of several groups of mucosal inflammation were done by one way Anova ( $F$ test) and LSD when there was a significance difference between means. Comparison between median were done by nonparametric test (Krurskal wallis-H test) followed by Mann-Whitney u test. Receiver operating curve characters were used to develop best cut off value in estimating the validity of different parameter in diagnosis of ulcerative colitis. Kappa measurement of agreement was done to test agreement between studied parameters and degree of mucosal inflammation. $\mathrm{P}$ value was considered significant when $\mathrm{P}$ value is less than 0.05 . 


\section{RESULTS}

Table (1): Demographics distribution among the examined patients

\begin{tabular}{|c|c|c|c|c|c|c|c|}
\hline \multirow{2}{*}{\multicolumn{2}{|c|}{$\begin{array}{c}\text { Degree of colonic mucosal } \\
\text { inflammation }\end{array}$}} & \multirow{2}{*}{ Number } & \multirow{2}{*}{$\begin{array}{l}\text { Sex } \\
(\mathrm{F} / \mathrm{M})\end{array}$} & \multicolumn{2}{|c|}{ Age } & \multirow{2}{*}{ f } & \multirow{2}{*}{$\mathbf{p}$} \\
\hline & & & & Mean \pm SD & Range & & \\
\hline Group B & $\begin{array}{l}\text { No mucosal } \\
\text { inflammation }\end{array}$ & 29 & $15 / 14$ & $32.2 \pm 9.6$ & $19-49$ & \multirow{4}{*}{0.32} & \multirow{4}{*}{0.81} \\
\hline \multirow{3}{*}{ Group A } & Mild & 6 & $3 / 3$ & $29.83 \pm 5.56$ & $25-39$ & & \\
\hline & Moderate & 9 & $7 / 2$ & $29.89 \pm 9.97$ & $\begin{array}{l}19-44 \\
\end{array}$ & & \\
\hline & Severe & 16 & $9 / 7$ & $29.63 \pm 10.52$ & $19-49$ & & \\
\hline
\end{tabular}

Table (2): Relation between degree of colonic mucosal inflammation SCCAI, endoscopic appearance and faecal calprotectin

\begin{tabular}{|c|c|c|c|c|}
\hline & \multicolumn{4}{|c|}{ Degree of colonic mucosal inflammation } \\
\hline & \multicolumn{2}{|c|}{ Group B } & \multicolumn{2}{|c|}{ Group A } \\
\hline & $\begin{array}{c}\text { No mucosal } \\
\text { inflammation }\end{array}$ & Mild & Moderate & Severe \\
\hline & $\begin{array}{ll}\text { Range } & \text { Media }\end{array}$ & Range & Range $\mathrm{Mec}$ & Range \\
\hline SCCAI & $(2-6)^{*}$ & $(6-11)$ & $(6-11)$ & $(4-12)$ \\
\hline Endoscopic appearance & $(0-1)^{*}$ & & & $(2-3$ \\
\hline $\begin{array}{c}\text { Fecal calprotectin } \\
(\mu \mathrm{g} / \mathrm{g})\end{array}$ & $(10-176)^{*}$ & $(10-176)$ & $(149-190)$ & $(170-215)$ \\
\hline
\end{tabular}

* Highly significant, $\mathbf{S C C A I}=$ Simple clinical colitis activity index

Table (3) Relation between degree of mucosal inflammation, CRP value and ESR

\begin{tabular}{|c|c|c|c|c|}
\hline & Group B & Group A & t & p \\
\hline CRP \pm SD & $\mathbf{6 . 7 6} \pm \mathbf{3 . 8 6}$ & $\mathbf{9 . 9 4} \pm \mathbf{5 . 0 9}$ & 2.711 & $0.009^{* *}$ \\
\hline ESR \pm SD & $\mathbf{2 3 . 1 4} \pm \mathbf{1 3 . 2 3}$ & $\mathbf{2 6 . 9 4} \pm \mathbf{1 0 . 3 6}$ & 1.242 & 0.219 \\
\hline
\end{tabular}

** Significant difference

Table (4): Validity of SCCAI, endoscopic appearance and faecal calprotectin in relation to pathology

\begin{tabular}{|l|c|c|c|c|}
\hline & Sensitivity & Specificity & $\begin{array}{c}\text { Positive } \\
\text { predictive value }\end{array}$ & $\begin{array}{c}\text { Negative } \\
\text { predictive value }\end{array}$ \\
\hline SCCAI & $83.9 \%$ & $72.4 \%$ & $76.5 \%$ & $80.8 \%$ \\
\hline $\begin{array}{l}\text { Endoscopic } \\
\text { appearance }\end{array}$ & $83.9 \%$ & $100 \%$ & $100 \%$ & $85.3 \%$ \\
\hline $\begin{array}{l}\text { Faecal } \\
\text { calprotectin }\end{array}$ & $93.5 \%$ & $89.7 \%$ & $90.6 \%$ & $92.9 \%$ \\
\hline
\end{tabular}




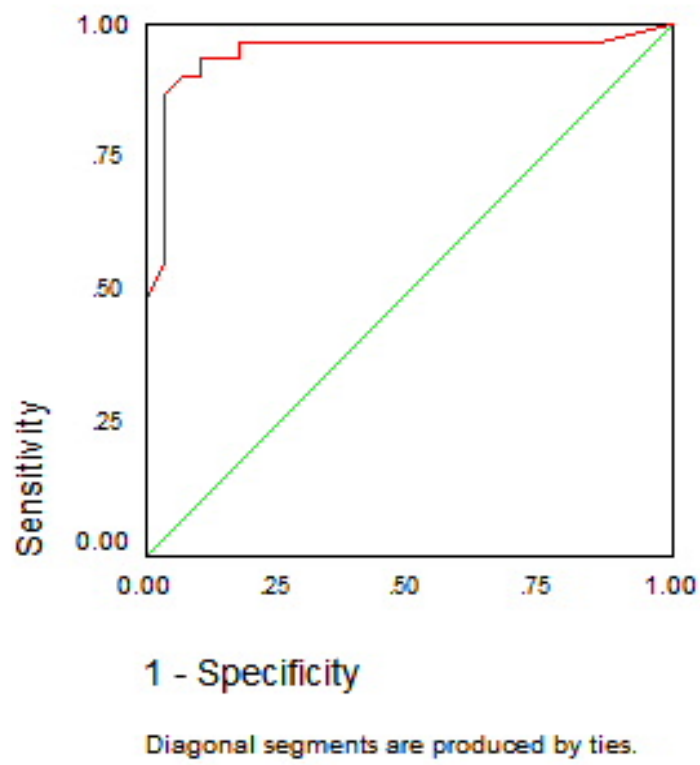

Fig (1): ROC curve of faecal calprotectin in predicting ulcerative colitis

\section{DISCUSSION}

Inflammatory bowel disease (IBD) and irritable bowel syndrome (IBS) are common entities. Both conditions may present with similar clinical features such as diarrhea and abdominal pain. Patients with IBD oscillate between periods of active and inactive disease and may even present with concomitant functional IBS [7]

Most patients with quiescent IBD have lowgrade inflammation and it is possible that symptomatic relapse occurs only when the inflammatory process reaches a critical intensity. Furthermore, because inflammation is a continuous process, direct assessment of the level of inflammatory activity may provide a quantitative presymptomatic measure of impending disease relapse [8].

Calprotectin is a valuable marker at the very early stage of inflammatory reactions in human beings [9].

Faecal calprotectin assessment is that it is a measure of mucosal inflammatory activity that may be detected at a level insufficient to cause an increase in ESR and CRP [10].

In the current study, more intense levels of inflammations are associated with elevated level of faecal calprotectin value, demonstrating a significant correlation between calprotectin and the severity of inflammation. Furthermore, faecal calprotectin had a high correlation with the histologic grading as that observed for endoscopy. Its sensitivity was $93.3 \%$, specificity was $89.7 \%$, and also it had a high negative predictive value. The results were the same as those obtained by Bunn et al., [6] who claimed that faecal calprotectin concentrations predicted the severity of colorectal inflammation, with advanced histological grades of colorectal inflammation.

Inflammation is the basis for many signs and symptoms of IBD, making its detection and monitoring fundamental to clinical management [5].

One means to assess inflammation that has been discussed in recent years is the analysis of the infiltration of neutrophil in the intestinal mucosa and their transmigration to the lumen [11].

Calprotectin is derived predominantly from neutrophils and, to a lesser extent, from monocytes and reactive macrophages [5].

Therefore the presence of calprotectin in faeces is directly proportional to neutrophil migration towards the intestinal tract [1].

When intestinal inflammation occurs, the calprotectin levels correlate closely with histological evaluation than macroscopic findings, suggesting that this biological marker is more sensible than endoscopy in evaluating IBDs activity [12]

Our study revealed that level of faecal calprotectin was higher in IBD patients than in non-IBD patients (by $205 \mu \mathrm{g} / \mathrm{g}$ ), which is matched by a study conducted by von Roon et al., [13] who stated that fecal calprotectin was 
higher in IBD patients than in non-IBD patients (by $219 \mu \mathrm{g} / \mathrm{g}$ ), and showed excellent pool sensitivity and specificity rates in distinguishing between these groups $(95 \%$ and $91 \%$, respectively).

In our study faecal calprotectin resulted the most accurate tool to assess the presence of active mucosal inflammation when compared to Creactive protein, erythrocyte sedimentation rate. These results had matched with Tibble et al. [10].

Our study showed that faecal calprotectin concentration above $72 \mu \mathrm{g} / \mathrm{g}$, gave a sensitivity of $93.5 \%$, a specificity of $89.7 \%$, a positive predictive value (PPV) of $90.6 \%$, and a negative predictive value (NPV) of $92.9 \%$ in predicting UC.

The data obtained by our study revealed that there is a good agreement between faecal calprotectin, and endoscopic appearance. These results showed that fecal calprotectin at a concentration above $72 \mu \mathrm{g} / \mathrm{g}$ was in agreement with Simple clinical colitis activity index when it was above 4 of about $46 \%$, while with endoscopy when the score above 1 the agreement was about $80 \%$.

Faecal calprotectin allows a non-invasive monitoring of disease activity, especially when the repeated measurements are considered, among UC patients, as better identifying controlled disease activity.

In most clinically quiescent IBD, residual mucosal inflammation is still present to some extent. When disease activity increases, clinical symptoms are usually not present during the early relapse stage. Faecal calprotectin seems to be able to detect subclinical mucosal inflammation, and thus might earlier identify those patients at risk for IBD relapse [14].

We can conclude that measurement of faecal calprotectin is highly useful for the diagnosis and disease monitoring of patients with ulcerative colitis, and might additionally predict disease outcome. It is a sensitive and direct biomarker of intestinal inflammation with a better performance than the traditional non-invasive tests. It is both easily carried out and reliable, which makes it suitable for use as a first-level test for the diagnosis of organic ulcerative colitis as well as for the activity monitoring of UC.
Funding: None .

\section{Conflicts of interest: None.}

Ethical approval: Informed consents were routinely obtained from patients. The study was performed in accordance with the ethical standards on human experimentation and with the Helsinki Declaration of 1964.

\section{REFERENCES}

1. Vermeire S, Van Assche G, Rutgeerts P. Laboratory markers in IBD: useful, magic, or unnecessary toys? Gut 2006;55: 426-31.

2. Bowles CJ, Leicester R, Romaya C, Swarbrick E, Williams CB, Epstein O.A prospective study of colonoscopy practice in the UK today: are we adequately prepared for national colorectal cancer screening tomorrow? Gut 2004; 53: 277-83.

3. Turner D, Walsh CM, Steinhart AH, Griffiths AM. Response to corticosteroids in severe ulcerative colitis: a systematic review of the literature and a meta-regression. Clin Gastroenterol Hepatol 2007;5:103-110.

4. Kaiser T, Langhorst J, Wittkowski H, Becker K, Friedrich AW, Rueffer A,et al. Faecal S100A12 as non-invasive marker distinguishing inflammatory bowel disease from irritable bowel syndrome. Gut 2007;56:1706-13.

5. Bjerke K, Halstensen TS, Jahnsen F, Pulford K, Brandtzaeg P. Distribution of macrophages and granulocytes expressing L1 protein (calprotectin) in human Peyer's patches compared with normal ileal lamina propria and mesenteric lymph nodes. Gut 1993;34: 1357-63.

6. Bunn SK, Bisset WM, Main MJ, Gray ES, Olson $\mathrm{S}$, Golden BE. Fecal calprotectin: validation as a non-invasive measure of bowel inflammation in childhood inflammatory bowel disease. $J$ Pediatr Gastroenterol Nutr 2001;33:14-22.

7. Spiller R, Aziz Q, Creed F, Emmanuel A, Houghton L, Hungin P, et al. Guidelines on the irritable bowel syndrome: mechanisms and practical management. Gut 2007; 56: 1770-98.

8. Tibble JA, Bjarnason I. Fecal calprotectin as an index of intestinal inflammation. Drugs Today (Barc) 2001;37:85-96.

9. Stockley RA, Dale I, Hill SL, Fagerhol MK. Relationship of neutrophil cytoplasmic protein (L1) to acute and chronic lung disease. Scand $J$ Clin Lab Invest 44: 629-634, 1984. 
10. Tibble JA, Sigthorsson G, Foster R, Forgacs I, Bjarnason I. Use of surrogate markers of inflammation and Rome criteria to distinguish organic from non-organic intestinal disease. Gastroenterology 2002;123 (2):450-60.

11. Silberer H, Küppers B, Mickisch O, Baniewicz W, Drescher M, Traber L, et al. Fecal leukocyte proteins in inflammatory bowel disease and irritable bowel syndrome. Clin Lab 2005;51:11726.

12. Limburg PJ, Ahlquist DA, Sandborn WJ, Mahoney DW, Devens ME, Harrington JJ, et al. Fecal calprotectin levels predict colorectal inflammation among patients with chronic diarrhea referred for colonoscopy. Am J Gastroenterol 2000;95:2831-7.

13. von Roon AC, Karamountzos L, Purkayastha S, Reese GE, Darzi AW, Teare JP, et al. Diagnostic precision of fecal calprotectin for inflam inflammatory bowel disease and colorectal malignancy. Am J Gastroenterol 2007;102:80313.

14. Emanuel Burria, Christoph Beglingera. Faecal calprotectin - a useful tool in the management of inflammatory bowel disease. Swiss Med Wkly. 2012;142:w13557. 